6. Real Decreto $1146 / 2006$, de 6 de octubre, por el que se regula la relación laboral especial de residencia para la formación de especialistas en ciencias de la Salud (BOE 7 de octubre de 2006).

7. San Román Terán CM, Alcalá-Zamora Salinas J, Guil García M, Fernández Sepúlveda S, Laín Guelvenzu JM, Peláez Domínguez S. Mala conducta científica en la comunicación de resultados biomédicos., ¿costumbre consagrada o laxitud de la ética? Rev Clin Esp 2004; 204: 393-7.

8. Maldonado Fernández M. Lenguaje medico, ética y medicina. Med Clin (Barc) 2004; 123: 262-4

9. Trilla A. Fraude científico: ¿presunción de inocencia? Med Clin (Barc) 1991; 96: 255-7.

10. Bravo Toledo R. Aspectos éticos de las publicaciones científicas. JANO 1997; 52: 74-6.

\section{Infección diseminada por Mycobacterium bovis tras instilación endovesical de BCG}

\section{Sr. Director:}

El bacilo de Calmette-Guerin (BCG) es una cepa viva atenuada de Mycobaterium bovis (M. bovis), especie del grupo Mycobacterium tuberculosis complex $(1,2)$. La administración de BCG intravesical es eficaz en el tratamiento del carcinoma superficial de vejiga (1-4). El mecanismo antitumoral parece ser una reacción de hipersensibilidad que causa una intensa infiltración por linfocitos y macrófagos, la cual facilita la eliminación de las células tumorales $(1,5,6)$. Aunque infrecuente, dicha terapia puede presentar efectos secundarios potencialmente letales. Presentamos el caso de una infección diseminada por M. bovis tras instilación endovesical de BCG.

Varón de 66 años con carcinoma transicional de vejiga que ingresa para tratamiento con $2^{\mathrm{a}}$ ciclo de BCG. A las 48 horas, comienza con fiebre persistente en picos, disuria, mal estado general e intensa astenia. En la analítica presentaba una discreta elevación de las transaminasas siendo la exploración y la radiología normales. Los cultivos, serologías de hepatitis, VIH, autoinmunidad, ecografía abdominal, BK en esputo y orina fueron negativos. Debido a la importancia de descartar infección diseminada por BCG se solicitaron scanner toracoabdominal que fue compatible con tuberculosis con diseminación miliar (Fig. 1) y gammagrafía con galio concordante con afectación tuberculosa multiorgánica (pulmones, riñones y glándula suprarrenales). Se realizó broncoscopia con lavado broncoalveolar para identificación de micobacterias que fue negativa y citología compatible con respuesta inflamatoria aguda. La biopsia transbronquial mostró infiltración granulomatosa no necrotizante. Se inició tratamiento con isoniazida, rifampicina y etambutol con mejoría parcial que fue completa al añadir corticoides.

Los efectos secundarios del tratamiento con BCG (BCGitis) suelen ser locales: cistitis $(91 \%)$, hematuria $(1 \%)$, prostatitis granulomatosa $(0,9 \%)$, espasmo vesical $(0,2 \%)$, obstrucción ureteral $(0,3 \%)(1-3,5,7,8)$. Existen complicaciones sistémicas que incluyen fiebre $(2,9 \%)$, rash $(0,3 \%)$, artralgia y artritis $(0,5 \%)$ y de mayor gravedad como neumonitis y/o hepatitis granulomatosa $(0,7 \%)$, sepsis $(0,4 \%)$, citopenia y/o absceso renal $(0,1 \%)(1-3,5,7,8) . \mathrm{La}$ etiopatogenia, diseminación BCG vs mecanismo de hipersensibilidad, permanece controvertida, aunque probablemente tenga un origen mixto (1-3,5). Datos a favor de un mecanismo de hipersensibilidad (tipo IV), son la alta frecuencia de la negatividad de los cultivos y la favorable respuesta a los corticoides. Esporádicamente en la

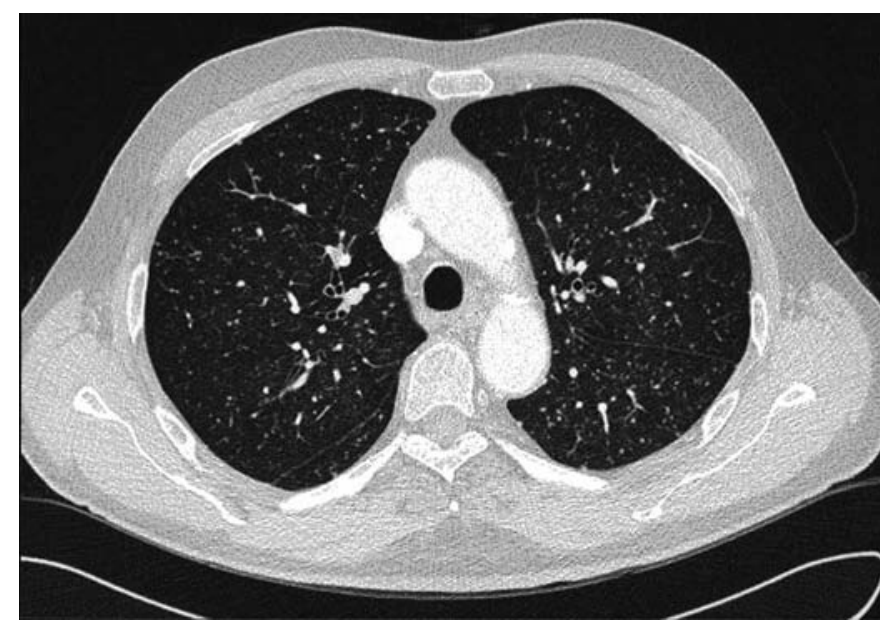

Fig. 1. TAC tórax: patrón micronodular bilateral con mayor afectación de los lóbulos superiores.

anatomía patológica se observan infiltrados de eosinófilos que también sugieren mecanismo de hipersensibilidad (2). Las hipótesis a favor de una diseminación infecciosa de BCG (siembra hematógena a partir de la vejiga) son la presencia, aunque ocasional, de tinción o cultivo de micobacterias, la presencia de granulomas no caseificantes en lugares distantes y la favorable respuesta a tuberculostáticos. El cultivo de micobacterias es el gold-standar (1), aunque con frecuencia hay falsos negativos por problemas en el procesamiento de las muestras (1) o porque se estima que se necesitan más de 10.000 organismos por gramo de tejido para que el cultivo sea positivo (2). La no demostración de micobacterias no descarta el diagnóstico que en muchas ocasiones es de probabilidad $(1,2,5)$. Hay relación temporal entre la clínica y los ciclos de BCG aunque a veces aparece tras años de tratamiento (2). Asimismo la alteración de la barrera urotelial por sondaje traumático favorece la sintomatología $(2,3,5)$. El uso de isoniazida como profilaxis previa al tratamiento con BCG no está justificado $(2,5)$. Destacar que en caso de fiebre que persiste tras 24 horas del tratamiento se recomienda suspender BCG y comenzar con isoniazida durante 3 meses $(1,2,7,9)$. La sepsis es la complicación más grave. En dicho caso es obligatorio suspender la administración de BCG y tratar con isoniazida más rifampicina más etambutol durante 6 meses, recomendándose el uso combinado de cicloserina (5 días) y de prednisolona $(1,4,5,7,9)$. $M$. bovis es resistente a pirazinamida $(2,5,7,9)$. Concluimos que ante todo paciente con afectación general tras BCG se debe descartar afectación sistémica por M. bovis. Con frecuencia el diagnóstico es de probabilidad ya que la demostración de micobacterias es difícil, no debiendo ésto condicionar el retraso en el tratamiento.

\section{I. de la Iglesia Fanjul, M. R. de Castro Losa, F. Mourad, T. Bajo Franco}

Servicio de Medicina Interna. Hospital de León. León

1. Del Castillo Durán Y, Santos Boda F, Castander Serentill D, Jubert Montaperto P, Espinosa Valencia P, Rabassó Sole C, et al. Tuberculosis miliar in a patient treated with intravesical instillations of bacillus Calmette-Guérin. Med Intensiva 2006; 30: 116-9.

2. Scully RE, Mark EJ, Mcneely WF, Ebeling SH. A 57 year-old man with fever and jaundice after intravesical instillation of bacilli CalmenteGuerin for bladder cancer. N Engl J Med 1998 17; 339: 831-7.

3. Gómez-Ferrer Lozano A, Navarro Antón JA, Sala Aznar A, Mola Arizo MJ, Gonzalvo Pérez V, Cantó Faubel E, et al. Neumonititis granuloma- 
tosa por instilación endovesical de BCG. Actas Uro Esp 2006; 30: 839842.

4. Lamm DL. Optimal BCG treatment of superficial bladder cancer as defined by American trials. Eur Uro 1992; 2: 12-6.

5. Martin Escudero JC, Pérez Paredes G, Ascensio Sánchez T, Herreros Fernández V. Neumonitis granulomatosa por BCG. An Med Interna (Madrid) 2003; 20: 57

6. Gupta RC, Lavengood R, Smith JP. Miliary tuberculosis due to intravesical Bacillus Calmette Guérin therapy. Chest 1998; 94: 1296-98.

7. Díaz C, Baldo C, Martín A, Fernández MJ, Muñoz M, Rodríguez L, et al. Tuberculosis parotidea secundaria a instilación vesical con BCG. Acta Otorrinolaringol Esp 2003; 54: 129-133.

8. Lamm DL, Vand der Meijden APM, Morales A, Brosman SA, Catalona WJ, Herr Hw, et al. Incidence and treatment of complications of bacillus Calmette-Guérin intravesical therapy in superficial bladder cancer. J Urol 1992; 147: 695-600.

9. Gilbert David N, Moellering Robert C, Sande Merle A. $19^{\text {a }}$ edition the Stanford Guide to antimicrobial therapy; 2006.

\section{Insuficiencia cardiaca de causa infecciosa}

\section{Sr. Director:}

La pericarditis purulenta es una rara localización infecciosa, pudiéndose producir por diseminación local o vía hematógenal. Siendo el absceso pericárdico una complicación excepcional de una pericarditis purulenta.

Presentamos un caso de un varón de 81 años de edad con intervención quirúrgica dos años antes de una valvulopatía aórtica con colocación de válvula protésica mecánica, posteriormente en clase funcional I de la New York Heart Association (NYHA), con ecocardiograma con válvula protésica normofuncionante sin otras alteraciones.

El paciente ingresa con una colecistitis gangrenada con plastrón perivesicular siendo intervenido de urgencia realizando una colecistectomía y limpieza quirúrgica del lecho vesicular, con cultivo del absceso peri-vesicular positivo para E. coli. El paciente presenta de forma progresiva mejoría del cuadro infeccioso y es dado de alta.

Reingresa varios días mas tarde con disnea y fiebre de $38,5^{\circ} \mathrm{C}$. En la exploración física se constata ingurgitación yugular, ruidos protésicos normofuncionantes y edema generalizado en situación de anasarca. Se inicia tratamiento depletivo con furosemida y antibiótico con imipenem. Se realiza hemocultivos seriados y una tomografía computerizada tóraco-abdominal (Fig. 1) constatando colección en lecho vesicular y subhepática de 4 x 6 x $5 \mathrm{~cm}$. Además se observó una colección paracardiaca pegada a ventrículo izquierdo de $5 \times 7 \mathrm{~cm}$ que comprimía el ventrículo izquierdo. En la ecocardiografía transtorácica se detecta una colección paracardiaca, con prótesis aortica normofuncionante sin evidencia de verrugas endomiocárdicas. Se realizó toracotomía de urgencia con drenaje de la colección intrapericárdica de aspecto serohemorrágico con cultivo positivo para E. coli, colocándose drenaje externo, además se realizó drenaje percutáneo de la colección intraabdominal. El paciente evolucionó satisfactoriamente desapareciendo la fiebre y resolviendose la situación de anasarca. Los hemocultivos fueron positivos para Bacteroides fragilis. Con el

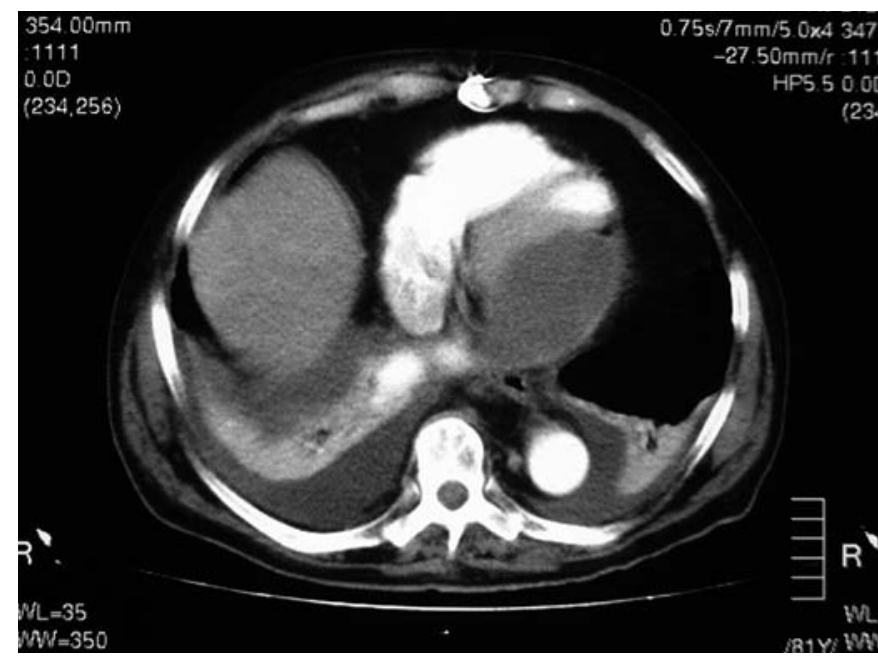

Fig. 1.

diagnóstico final de absceso pericardico por $E$. coli secundario a absceso intraabdominal.

En nuestro caso la infección por E. coli se produjo por diseminación local a través del diafragma cultivándose secuencialmente en absceso abdominal y pericárdico. Es plausible, que como ocurria en nuestro paciente, la cirugía cardiaca pueda dar lugar a formación de adherencias pericárdicas que favorezcan esta manifestación de debut (2). Las dos manifestaciones principales de los abscesos pericárdicos son la insuficiencia cardiaca restrictiva (especialmente en abscesos localizados adyacentes a aurículas o ventrículo derecho, y más difícil cuando lo hacen sobre ventrículo izquierdo) y el cuadro séptico asociado $(3,4)$. Nuestro paciente presentaba un absceso pegado a la pared libre del ventrículo izquierdo que comprimía hasta casi colapsar al ventrículo en diástole originándole insuficiencia cardiaca grave con situación de anasarca y cuadro séptico asociado. El tratamiento pasa siempre por un abordaje diagnóstico y terapéutico con punción o drenaje quirúrgico abierto y tratamiento antibiótico según el gérmen aislado. En nuestro caso además se realizó un drenaje percutáneo de la colección intraabdominal con resolución del cuadro infeccioso y resolución de la insuficiencia cardiaca.

\section{Fuentes Pardo, M. Belhassen García, J. Pardo Lledías, A. Carpio Pérez, I. Cruz ${ }^{1}$}

Servicios de Medicina Interna III y de ${ }^{I}$ Cardiología. Hospital Universitario. Salamanca

1. Nwiloh JO, Egbe PA, Tagoe AT, Weaver LW. Staphylococcus aureus Pericarditis Masquerading as Anterior Mediastinal:mediastinal mass from pericarditis. Chest 2000; 118; 1832-1833.

2. Mitsuma W, Ito M, Fujita S, Tsuchida K, Takahashi M, Hayashi J, Kodama M, Aizawa Y. Pericardial abscess detected by gallium- 67 scintigraphy 40 years after cardiac surgery. Int Heart J 2005; 46: 1119-22.

3. Caliskan K, Galema TW, Klootwijk AP. Tuberculous pericardial abscess causing right ventricular obstruction. Heart 2004; 90: 1041.

4. Hegde AN, Desai SB, Shivdasani B. Tuberculous pericardial abscess Eur J Cardiothorac Surg 2005; 28: 166. 